\title{
ON THE ESSENTIAL COMMUTANT OF $\mathcal{T}(\mathrm{QC})$
}

\author{
JINGBO XIA
}

\begin{abstract}
Let $\mathcal{T}(\mathrm{QC})$ (resp. $\mathcal{T}$ ) be the $C^{*}$-algebra generated by the Toeplitz operators $\left\{T_{\varphi}: \varphi \in \mathrm{QC}\right\}$ (resp. $\left\{T_{\varphi}: \varphi \in L^{\infty}\right\}$ ) on the Hardy space $H^{2}$ of the unit circle. A well-known theorem of Davidson asserts that $\mathcal{T}(\mathrm{QC})$ is the essential commutant of $\mathcal{T}$. We show that the essential commutant of $\mathcal{T}(\mathrm{QC})$ is strictly larger than $\mathcal{T}$. Thus the image of $\mathcal{T}$ in the Calkin algebra does not satisfy the double commutant relation. We also give a criterion for membership in the essential commutant of $\mathcal{T}(\mathrm{QC})$.
\end{abstract}

\section{INTRODUCTION}

Let $H$ be a separable, infinite-dimensional Hilbert space. Recall that the Calkin algebra $\mathcal{Q}$ is defined to be the quotient algebra $\mathcal{B}(H) / \mathcal{K}(H)$, where $\mathcal{B}(H)$ (resp. $\mathcal{K}(H)$ ) is the collection of bounded (resp. compact) operators on $H$. The essential commutant of a subset $G$ of $\mathcal{B}(H)$ is defined to be

$$
\operatorname{Ess} \operatorname{Com}(G)=\{X \in \mathcal{B}(H):[T, X] \in \mathcal{K}(H) \text { for every } T \in G\} .
$$

Let $\pi: \mathcal{B}(H) \rightarrow \mathcal{Q}$ be the quotient map. Then $\pi(\operatorname{EssCom}(G))$ is the commutant of $\pi(G)$ in $\mathcal{Q}$. That is, $\{\pi(G)\}^{\prime}=\pi(\operatorname{Ess} \operatorname{Com}(G))$. For the discussion below, all algebras and subalgebras are assumed to be unital.

A well-known result of Voiculescu asserts that every separable $C^{*}$-subalgebra $\mathcal{A}$ of $\mathcal{Q}$ satisfies the double commutant relation $\mathcal{A}=\mathcal{A}^{\prime \prime}$ [13]. An equally well-known result due to Johnson and Parrott [7] and Popa [11] tells us that if $B$ is a von Neumann algebra on $H$, then $\operatorname{EssCom}(B)=B^{\prime}+\mathcal{K}(H)$. Thus if $\mathcal{A}$ is the image of a von Neumann algebra in $\mathcal{Q}$, then it also satisfies the double commutant relation $\mathcal{A}=\mathcal{A}^{\prime \prime}$.

On the other hand, there exist non-separable $C^{*}$-subalgebras of $\mathcal{Q}$ for which the double commutant relation fails. Indeed Example 2.4 in the Johnson-Parrott paper [7] provides just such an example. In [1], Berger and Coburn gave an example of a simple $C^{*}$-subalgera of $\mathcal{Q}$ which does not satisfy the double commutant relation. Subsequently, the author showed that $\mathcal{A} \neq \mathcal{A}^{\prime \prime}$ is quite common among $C^{*}$-subalgebras of $\mathcal{Q}$ in the following sense: If $B$ is a von Neumann algebra and if the dimension of $B$ as a linear space is infinite, then $B$ contains a $C^{*}$-subalgebra $A$ such that $\pi(A) \neq \pi(B)$ and $\{\pi(A)\}^{\prime \prime}=\pi(B)[15]$.

Given these positive and negative results about the double commutant relation, for any $C^{*}$-subalgebra of $\mathcal{Q}$ which is of any interest but not covered by these general results, it seems to be natual to ask whether or not it satisfies the double commutant

Received by the editors January 1, 2005 and, in revised form, May 8, 2006.

2000 Mathematics Subject Classification. Primary 42A38, 46L05, 47L80.

This work was supported in part by National Science Foundation grant DMS-0100249. 
relation. Such a problem usually boils down to the determination of the essential commutant of a specific $C^{*}$-algebra $[1,4,7,11,15]$. The focus of this paper is on a specific essential commutant on the Hardy space $H^{2}$ of the unit circle $\mathbf{T}=\{\tau \in$ $\mathbf{C}:|\tau|=1\}$. This turns out to be one place where harmonic analysis finds a nice application in the theory of operator algebras.

Recall that the Hardy space $H^{2}$ is the closure of the linear span of $\left\{e^{i n t}: n=0\right.$, $1,2, \ldots\}$ in $L^{2}$. Let $P: L^{2} \rightarrow H^{2}$ be the orthgonal projection. Given a $\varphi \in L^{\infty}$, the Toeplitz operator $T_{\varphi}$ is defined by the formula $T_{\varphi} g=P \varphi g, g \in H^{2}$. Let

$$
\mathcal{T}=\text { the } C^{*} \text {-algebra generated by }\left\{T_{\varphi}: \varphi \in L^{\infty}\right\},
$$

which is commonly referred to as the full Toeplitz algebra.

Let $\mathrm{QC}=\left(H^{\infty}+C\right) \cap \overline{\left(H^{\infty}+C\right)}$, the collection of quasi-continuous functions on the unit circle. It is well known that $\mathrm{QC}=\mathrm{VMO} \cap L^{\infty}[5$, page 377]. Define

$$
\mathcal{T}(\mathrm{QC})=\text { the } C^{*} \text {-algebra generated by }\left\{T_{\varphi}: \varphi \in \mathrm{QC}\right\} \text {. }
$$

A key motivating factor for this investigation is the following famous result:

Theorem 1.1 (Davidson [4]). The essential commutant of $\mathcal{T}$ equals $\mathcal{T}(Q C)$.

An immediate consequence of Theorem 1.1 is that $\pi(\mathcal{T}(\mathrm{QC}))$ satisfies the double commutant relation in $\mathcal{Q}$. On the other hand, although more than a quarter of a century has passed since Davidson's theorem was published, little is known about $\operatorname{EssCom}(\mathcal{T}(\mathrm{QC}))$. Given Theorem 1.1, it seems natural to ask, what is $\operatorname{Ess} \operatorname{Com}(\mathcal{T}(\mathrm{QC}))$ ? In particular, does $\operatorname{Ess} \operatorname{Com}(\mathcal{T}(\mathrm{QC}))$ coincide with $\mathcal{T}$ ? See [14, Problem 13]. We now have an answer:

Theorem 1.2. The essential commutant of $\mathcal{T}(Q C)$ is strictly larger than $\mathcal{T}$.

Given Theorem 1.1, Theorem 1.2 is obviously equivalent to

Theorem 1.3. The image of $\mathcal{T}$ in the Calkin algebra does not satisfy the double commutant relation.

This seems to be all the more interesting if we consider the following fact: Let $\mathcal{T}\left(H^{\infty}+C\right)$ be the (non-self-adjoint) operator algebra generated by $\left\{T_{\varphi}: \varphi \in\right.$ $\left.H^{\infty}+C\right\}$. Davidson showed in [4] that $\pi\left(\mathcal{T}\left(H^{\infty}+C\right)\right)$ is a maximal abelian algebra in the Calkin algebra $\mathcal{Q}$. It is well known that $\mathcal{T}\left(H^{\infty}+C\right)$ generates $\mathcal{T}$ as a $C^{*}$-algebra.

As we will see in the proof of Theorem 1.2, $\operatorname{Ess} \operatorname{Com}(\mathcal{T}(\mathrm{QC}))$ contains operators which seem to defy general description. Nevertheless, $\operatorname{Ess} \operatorname{Com}(\mathcal{T}(\mathrm{QC}))$ admits a characterization in terms of a BMO-related continuity.

Theorem 1.4. Let $A$ be a bounded operator on $H^{2}$ such that $\left[T_{h}, A\right]$ is compact for every continuous function $h$ on the unit circle. Then $A$ belongs to the essential commutant of $\mathcal{T}(Q C)$ if and only if $A$ has the following property: For every $\epsilon>0$, there exists a $\delta=\delta(\epsilon)>0$ such that the inequality $\left\|\left[T_{\varphi}, A\right]\right\| \leq \epsilon$ holds for every $\varphi$ in $L^{\infty}$ satisfying the conditions $\|\varphi\|_{B M O} \leq \delta$ and $\|\varphi\|_{\infty} \leq 1$.

The rest of the paper consists of the proofs of these results. More specifically, we will prove Theorem 1.2 in Section 2 and Theorem 1.4 in Section 3. The proof of Theorem 1.4 uses techniques and ideas similar to those in $[4,7]$.

Although Theorem 1.2 was motivated by $C^{*}$-algebraic considerations, the proof we present here is based on harmonic analysis. In particular, the proof requires 
the full strength of the theory of Calderón-Zygmund operators $[2,3,12]$. The most difficult step in the proof is a norm estimate $\left\|\left[M_{f}, T\right]\right\| \leq C\|f\|_{\text {BMO }}$. This involves hard analysis, but fortunately we can use existing results $[2,3,8]$ to establish the required estimate. Thus the task we actually carry out in the proof of Theorem 1.2 is relatively easy.

\section{Proof of Theorem 1.2}

The first step in the proof is to transform the problem from the unit circle $\mathbf{T}$ to the real line $\mathbf{R}$. In this section $d m$ stands for the Lebesgue measure on $\mathbf{R}$. Let $H^{2}(\mathbf{R})$ be the Hardy space for the upper-half plane. We denote the orthogonal projection from $L^{2}(\mathbf{R})$ to $H^{2}(\mathbf{R})$ by $P_{W}$. The purpose of the subscript $W$, which stands for Wiener-Hopf, is to distinguish $P_{W}$ from the Hardy projection $P$ on the unit circle. For any $f \in L^{\infty}(\mathbf{R})$, the operator $W_{f}=P_{W} M_{f} \mid H^{2}(\mathbf{R})$ will be called a Wiener-Hopf operator, again to distinguish it from the unit circle case. Let $\mathcal{W}$ be the $C^{*}$-algebra generated by $\left\{W_{f}: f \in L^{\infty}(\mathbf{R})\right\}$.

Consider the conformal mapping

$$
\alpha(z)=\frac{i-z}{i+z}
$$

from the upper-half plane to the unit disc. The formula

$$
(U g)(x)=\frac{1}{\sqrt{\pi}} \cdot \frac{1}{i+x} g\left(\frac{i-x}{i+x}\right), \quad g \in L^{2}(\mathbf{T}),
$$

defines a unitary operator from $L^{2}(\mathbf{T})$ to $L^{2}(\mathbf{R})$. The coefficient $1 / \sqrt{\pi}$ in the above is due to the normalization of the norms on these spaces: As usual, we define $\|g\|^{2}=(1 / 2 \pi) \int_{-\pi}^{\pi}\left|g\left(e^{i \theta}\right)\right|^{2} d \theta$ for $g \in L^{2}(\mathbf{T})$ and $\|G\|^{2}=\int|G(x)|^{2} d x$ for $G \in L^{2}(\mathbf{R})$. We have

$$
U H^{2}=H^{2}(\mathbf{R}) \quad \text { and } \quad U P U^{*}=P_{W} .
$$

Thus $U T_{\varphi} U^{*}=W_{\varphi \circ \alpha}$ for every $\varphi \in L^{\infty}$. Therefore

$$
U \mathcal{T} U^{*}=\mathcal{W} .
$$

Thus our goal is to construct a bounded operator on $H^{2}(\mathbf{R})$ which commutes with $\left\{W_{\varphi \circ \alpha}: \varphi \in \mathrm{QC}\right\}$ modulo compact operators and which does not belong to $\mathcal{W}$.

To construct such an operator, we pick a $u \in C_{c}^{\infty}[0, \infty)$ such that $u=1$ on $[7 / 10,4 / 5], u=0$ on $[0,3 / 5] \cup[9 / 10, \infty)$, and $0 \leq u \leq 1$ on the rest of $[0, \infty)$. Define

$$
\xi(x)=\left\{\begin{array}{ccc}
u(x) & \text { if } & x \geq 0, \\
-u(|x|) & \text { if } & x<0 .
\end{array}\right.
$$

The choice of $u$ ensures that $\xi \in C_{c}^{\infty}(\mathbf{R})$. Let $\hat{\xi}$ denote the Fourier transform of $\xi$, i.e.,

$$
\hat{\xi}(\lambda)=\frac{1}{\sqrt{2 \pi}} \int \xi(x) e^{-i \lambda x} d x .
$$

Then $\hat{\xi}$ is a smooth, rapidly decreasing function on $\mathbf{R}$. Since $\xi(-x)=-\xi(x)$, we also have $\hat{\xi}(-\lambda)=-\hat{\xi}(\lambda)$. Thus we can pick an $\omega>0$ such that

$$
\hat{\xi}(\omega) \neq 0 \text {. }
$$


Furthermore, the property $\xi(-x)=-\xi(x)$ implies that

$$
\hat{\xi}(0)=\frac{1}{\sqrt{2 \pi}} \int \xi(x) d x=0 .
$$

For each $m \in \mathbf{N}$, define the function

$$
\xi_{m}(x)=2^{m} \xi\left(2^{m} x\right), \quad x \in \mathbf{R} .
$$

Let $\hat{\xi}_{m}$ be the Fourier transform of $\xi_{m}$. Then $\hat{\xi}_{m}$ satisfies the relation

$$
\hat{\xi}_{m}(\lambda)=\hat{\xi}\left(2^{-m} \lambda\right), \quad \lambda \in \mathbf{R} .
$$

Next we inductively select a strictly increasing sequence $\left\{\lambda_{j}\right\}_{j=0}^{\infty}$ of non-negative real numbers and a strictly increasing sequence $\left\{m_{j}\right\}_{j=1}^{\infty}$ of positive integers as follows: We begin with $\lambda_{0}=0$. Pick any $m_{1} \in \mathbf{N}$ such that $2^{m_{1}} \omega-1>\lambda_{0}$. Suppose that $j \geq 1$ and that we have selected $\lambda_{0}<\ldots<\lambda_{j-1}$ and $m_{1}<\ldots<m_{j}$ such that $2^{m_{j}} \omega-j>\lambda_{j-1}$. Since $\lim _{|\lambda| \rightarrow \infty}|\hat{\xi}(\lambda)|=0$, there exists a $\lambda_{j}>2^{m_{j}} \omega+j$ such that

$$
\left|\hat{\xi}_{m_{1}}(\lambda)\right|+\ldots+\left|\hat{\xi}_{m_{j}}(\lambda)\right| \leq 2^{-j-1} \quad \text { for all }|\lambda| \geq \lambda_{j}
$$

Set

$$
\mu_{j}=\lambda_{j}+2 j
$$

Since $\hat{\xi}$ is continuous, by (2.3), we can pick an integer $m_{j+1}>m_{j}$ such that $2^{m_{j+1}} \omega-(j+1)>\lambda_{j}$ and such that

$$
\left|\hat{\xi}_{m_{j+1}}(\lambda)\right|=\left|\hat{\xi}\left(2^{-m_{j+1}} \lambda\right)\right| \leq 2^{-j-1} \quad \text { if }|\lambda| \leq \mu_{j}
$$

Thus we have inductively defined $\left\{\lambda_{j}\right\}_{j=0}^{\infty}$ and $\left\{m_{j}\right\}_{j=1}^{\infty}$.

For each $n \in \mathbf{N}$ consider the function

$$
K_{n}=\sum_{j=1}^{n} \xi_{m_{j}}
$$

and the convolution operator

$$
\left(T_{n} g\right)(x)=\int K_{n}(x-y) g(y) d y, \quad g \in L^{2}(\mathbf{R}) .
$$

Let $\hat{K}_{n}$ be the Fourier transform of $K_{n}$. By (2.5) and (2.7), if $\lambda_{j-1} \leq|\lambda|<\lambda_{j}$, $j \geq 1$, then $\left|\hat{K}_{n}(\lambda)\right| \leq\left\|\hat{\xi}_{m_{j}}\right\|_{\infty}+\sum_{k \geq j} 2^{-k}$. Since $\left\|\hat{\xi}_{m_{j}}\right\|_{\infty}=\|\hat{\xi}\|_{\infty}$ (see $\left.(2.4)\right)$, we have

$$
\left\|\hat{K}_{n}\right\|_{\infty} \leq\|\hat{\xi}\|_{\infty}+1
$$

$n \in \mathbf{N}$. Define the function

$$
\hat{K}(\lambda)=\lim _{n \rightarrow \infty} \hat{K}_{n}(\lambda)=\sum_{j=1}^{\infty} \hat{\xi}_{m_{j}}(\lambda), \quad \lambda \in \mathbf{R},
$$

where the existence of the limit is guaranteed by (2.7). By (2.8), $\|\hat{K}\|_{\infty} \leq\|\hat{\xi}\|_{\infty}+1$. The Fourier transform provides the unitary equivalence between $T_{n}$ and $M_{\sqrt{2 \pi} \hat{K}_{n}}$, the operator of multiplication by $\sqrt{2 \pi} \hat{K}_{n}$. Therefore the strong limit

$$
T=\mathrm{s}-\lim _{n \rightarrow \infty} T_{n}
$$


exists. Furthermore, (2.8) implies $\|T\| \leq \sqrt{2 \pi}\left(\|\hat{\xi}\|_{\infty}+1\right)$. Let

$$
K=\sum_{j=1}^{\infty} \xi_{m_{j}} .
$$

Since $\xi_{m}(x)=2^{m} \xi\left(2^{m} x\right)$, we have $\xi_{m}^{\prime}(x)=2^{2 m} \xi^{\prime}\left(2^{m} x\right)$ for each $m \in \mathbf{N}$. The supports of $\xi_{m}$ and $\xi_{m}^{\prime}$ are strictly contained in $\left(-2^{-m},-2^{-m-1}\right) \cup\left(2^{-m-1}, 2^{-m}\right)$. Since the sequence $\left\{m_{j}\right\}_{j=1}^{\infty}$ is strictly increasing, this gives us

$$
\begin{aligned}
\left|\frac{d}{d x} K(x)\right| & \leq\left\|\xi^{\prime}\right\|_{\infty} x^{-2} \text { and } \\
|K(x)| & \leq|x|^{-1}
\end{aligned}
$$

for all $x \neq 0$. Thus $T$ is a Calderón-Zygmund operator in the sense that

$$
(T g)(x)=\int K(x-y) g(y) d y
$$

for $g \in L^{2}(\mathbf{R})$ and $x$ not in the support of $g$. The key to the proof of Theorem 1.2 is the following weighted norm inequality of Coifman and Fefferman [2].

Proposition 2.1. Let $w$ be a weight function on $\mathbf{R}$ which satisfies the $\left(A_{2}\right)$ condition

$$
\frac{1}{|I|} \int_{I} w d m \frac{1}{|I|} \int_{I} w^{-1} d m \leq L<\infty
$$

for every interval $I \subset \mathbf{R}$ with $0<|I|<\infty$. Then there exists a constant $N$ which depends only on the bounds in (2.9) and (2.10), $\|T\|$, and the $\left(A_{2}\right)$-bound $L$ above such that

$$
\int|T g|^{2} w d m \leq N \int|g|^{2} w d m, \quad g \in L^{2}(\mathbf{R}) .
$$

This proposition is an immediate consequence of Theorem I, Theorem III and Lemma 3 in [2]. Using the John-Nirenberg Theorem, from the above weighted norm inequality one can derive an estimate for commutators in terms of the BMO norm.

Lemma 2.2. There exists a constant $C_{2.2}$ such that the inequality

$$
\left\|\left[M_{f}, T\right]\right\| \leq C_{2.2}\|f\|_{B M O}
$$

holds for all $f \in L^{\infty}(\mathbf{R})$.

Proof. This type of estimate is actually well known. See, for example, [3, pages 620-621] or [10, Theorem 4.2]. Nevertheless, we reproduce the details of the proof here for the benefit of those readers who are not familiar with weighted norm inequalities.

For an interval $I \subset \mathbf{R}$, we write $\varphi_{I}=\int_{I} \varphi d m /|I|$ as usual. By the John-Nirenberg Theorem, there are universal constants $C$ and $c$ such that

$$
\frac{1}{|I|} \int_{I} e^{t\left|\psi-\psi_{I}\right|} d m \leq 1+C \quad \text { if } t \leq \frac{c}{2\|\psi\|_{\text {BMO }}}
$$

for all $\psi \in \operatorname{BMO}(\mathbf{R})$ and all intervals $I \subset \mathbf{R}$ [5, Theorem VI.2.1]. To prove the lemma, it suffices to consider real-valued $f \in L^{\infty}(\mathbf{R})$. For such an $f$ it follows from (2.11) that

$$
\frac{1}{|I|} \int_{I} e^{t f} d m \frac{1}{|I|} \int_{I} e^{-t f} d m \leq(1+C)^{2}
$$


for real $t$ satisfying the condition $|t| \leq c\left(2\|f\|_{\mathrm{BMO}}\right)^{-1}$. If we consider $e^{t f}$ as a weight function, then $(2.12)$ is an $\left(A_{2}\right)$-condition. Thus, by Proposition 2.1, there is a constant $C_{1}$ which depends only on $\|T\|$ and the bounds in (2.9), (2.10) and (2.12) such that

$$
\int|T g|^{2} e^{t f} d m \leq C_{1} \int|g|^{2} e^{t f} d m
$$

for all $g \in L^{2}(\mathbf{R})$ and $|t| \leq c\left(2\|f\|_{\mathrm{BMO}}\right)^{-1}$. Note that the norm of $M_{w^{1 / 2}} T M_{w^{-1 / 2}}$ on the unweighted space $L^{2}(\mathbf{R})$ equals the norm of $T$ on the weighted space $L^{2}(\mathbf{R}, w d m)$. Therefore from (2.13) we obtain the estimate

$$
\left\|e^{t f} T e^{-t f} g\right\| \leq C_{1}^{1 / 2}\|g\| \quad \text { for } g \in L^{2}(\mathbf{R}) \text { and }|t| \leq \frac{c}{4\|f\|_{\mathrm{BMO}}} .
$$

It is easy to see that for any $\eta, h \in L^{2}(\mathbf{R})$, the function

$$
z \mapsto\left\langle e^{z f} T e^{-z f} \eta, h\right\rangle
$$

is analytic on $\mathbf{C}$. For $z \in \mathbf{C}$ with $|z| \leq c\left(4\|f\|_{\mathrm{BMO}}\right)^{-1}$, (2.14) holds for $t=\operatorname{Re}(z)$. Since $f$ is real valued, we have $\left\|e^{z f} T e^{-z f} \eta\right\|=\left\|e^{\operatorname{Re}(z) f} T e^{-z f} \eta\right\|$ and $\left\|e^{-i \operatorname{Im}(z) f} \eta\right\|=$ $\|\eta\|$. Hence it follows from (2.14) that

$$
\left\|e^{z f} T e^{-z f} \eta\right\| \leq C_{1}^{1 / 2}\|\eta\|
$$

for $z \in \mathbf{C}$ with $|z| \leq c\left(4\|f\|_{\mathrm{BMO}}\right)^{-1}$. Now set $r(f)=c\left(4\|f\|_{\mathrm{BMO}}\right)^{-1}$. Then

$$
\left\langle\left[M_{f}, T\right] \eta, h\right\rangle=\left.\frac{d}{d z}\left\langle e^{z f} T e^{-z f} \eta, h\right\rangle\right|_{z=0}=\frac{1}{2 \pi i} \int_{|z|=r(f)} \frac{1}{z^{2}}\left\langle e^{z f} T e^{-z f} \eta, h\right\rangle d z .
$$

Combining this with (2.15), we have

$$
\left|\left\langle\left[M_{f}, T\right] \eta, h\right\rangle\right| \leq \frac{1}{r(f)} \max _{|z|=r(f)}\left|\left\langle e^{z f} T e^{-z f} \eta, h\right\rangle\right| \leq \frac{4\|f\|_{\mathrm{BMO}}}{c} C_{1}^{1 / 2}\|\eta\|\|h\| .
$$

This gives us the estimate $\left\|\left[M_{f}, T\right]\right\| \leq 4 C_{1}^{1 / 2} c^{-1}\|f\|_{\text {BMO }}$.

Finally, we define the operator $B$ by the formula

$$
B g=P_{W} M_{\chi_{[0,1]}} T M_{\chi_{[0,1]}} g, \quad g \in H^{2}(\mathbf{R}) .
$$

Recalling (2.1), the proof of Theorem 1.2 will be complete once we show

(a) $\left[W_{\varphi \circ \alpha}, B\right]$ is compact for every $\varphi \in \mathrm{QC}$;

(b) $B \notin \mathcal{W}$.

If $\varphi \in \mathrm{QC}$, then $\left[M_{\varphi \circ \alpha}, P_{W}\right]=U\left[M_{\varphi}, P\right] U^{*}$ is compact. Thus, if $\varphi \in \mathrm{QC}$, then $P_{W} M_{\varphi \circ \alpha}\left(1-P_{W}\right)$ and $\left(1-P_{W}\right) M_{\varphi \circ \alpha} P_{W}$ are compact. It is straightforward to verify that if $\varphi \in \mathrm{QC}$, then $\varphi \circ \alpha \in \operatorname{VMO}(\mathbf{R}) \cap L^{\infty}(\mathbf{R})$. Hence (a) follows from

Lemma 2.3. If $f \in V M O(\mathbf{R}) \cap L^{\infty}(\mathbf{R})$, then the operator $M_{\chi_{[0,1]}}\left[M_{f}, T\right] M_{\chi_{[0,1]}}$ is compact.

Proof. First let us consider $f$ which satisfies a Lipschitz condition

$$
|f(x)-f(y)| \leq L|x-y|
$$

for $x, y \in[0,1]$. Recalling $(2.10)$, the kernel of the operator $M_{\chi_{[0,1]}}\left[M_{f}, T\right] M_{\chi_{[0,1]}}$ satisfies the estimate

$$
\left|\chi_{[0,1]}(x)(f(x)-f(y)) K(x-y) \chi_{[0,1]}(y)\right| \leq L, \quad x, y \in[0,1] .
$$


Since the kernel is supported on $[0,1] \times[0,1]$, we have $\left\|M_{\chi_{[0,1]}}\left[M_{f}, T\right] M_{\chi_{[0,1]}}\right\|_{2} \leq L$, where $\|\cdot\|_{2}$ denotes the Hilbert-Schmidt norm. In particular, $M_{\chi_{[0,1]}}\left[M_{f}, T\right] M_{\chi_{[0,1]}}$ is compact if $f$ satisfies a Lipschitz condition. By the usual approximation, $M_{\chi_{[0,1]}}\left[M_{f}, T\right] M_{\chi_{[0,1]}}$ is also compact if $f \in C_{b}(\mathbf{R})$, where $C_{b}(\mathbf{R})$ denotes the collection of bounded continuous functions on $\mathbf{R}$. Finally, let $f \in \operatorname{VMO}(\mathbf{R}) \cap L^{\infty}(\mathbf{R})$. Then by a theorem of Sarason (see [5, Theorem VI.5.1]) there is a sequence $\left\{f_{k}\right\} \subset$ $C_{b}(\mathbf{R})$ such that $\lim _{k \rightarrow \infty}\left\|f-f_{k}\right\|_{\text {BMO }}=0$. It follows from Lemma 2.2 and the compactness of $M_{\chi_{[0,1]}}\left[M_{f_{k}}, T\right] M_{\chi_{[0,1]}}$ that $M_{\chi_{[0,1]}}\left[M_{f}, T\right] M_{\chi_{[0,1]}}$ is compact.

We now turn to the proof of (b), which is easy given what has been built into the selection of $\xi,\left\{\lambda_{j}\right\}_{j=0}^{\infty}$ and $\left\{m_{j}\right\}_{j=1}^{\infty}$. For each $\lambda \in \mathbf{R}$, define the unitary operator

$$
\left(V_{\lambda} g\right)(x)=e^{i \lambda x} g(x), \quad g \in L^{2}(\mathbf{R}) .
$$

Under the Fourier transform, $V_{\lambda}^{*} P_{W} V_{\lambda}$ is unitarily equivalent to the orthogonal projection from $L^{2}(\mathbf{R})$ onto the subspace $\left\{g \in L^{2}(\mathbf{R}): g=0\right.$ on $\left.(-\infty,-\lambda)\right\}$. Hence

$$
\text { S- } \lim _{\lambda \rightarrow \infty} V_{\lambda}^{*} P_{W} V_{\lambda}=1 .
$$

For each $\lambda \in \mathbf{R}$, define $\tilde{V}_{\lambda}=P_{W} V_{\lambda} \mid H^{2}(\mathbf{R})$, which is the compression of $V_{\lambda}$ to the subspace $H^{2}(\mathbf{R})$. By $(2.16)$ and the identity $V_{\lambda}^{*} M_{f} V_{\lambda}=M_{f}, f \in L^{\infty}(\mathbf{R})$, the strong limit

$$
s(A)=\mathrm{s}-\lim _{\lambda \rightarrow \infty} \tilde{V}_{\lambda}^{*} A \tilde{V}_{\lambda}
$$

exists for every $A \in \mathcal{W}$. Thus (b) follows from

Lemma 2.4. Let $g$ be a non-zero vector in the Hilbert space $H^{2}(\mathbf{R})$. Then

$$
\limsup _{\lambda \rightarrow \infty}\left|\left\langle\tilde{V}_{\lambda}^{*} B \tilde{V}_{\lambda} g, g\right\rangle\right|>0
$$

and

$$
\liminf _{\lambda \rightarrow \infty}\left|\left\langle\tilde{V}_{\lambda}^{*} B \tilde{V}_{\lambda} g, g\right\rangle\right|=0
$$

Proof. Let $\psi=\chi_{[0,1]} g$ and let $\hat{\psi}$ be the Fourier transform of $\psi$. Suppose that $\lambda>0$. Then $H^{2}(\mathbf{R})$ is invariant under $V_{\lambda}$. Thus

$$
\begin{aligned}
\left\langle\tilde{V}_{\lambda}^{*} B \tilde{V}_{\lambda} g, g\right\rangle & =\left\langle M_{\chi_{[0,1]}} T M_{\chi_{[0,1]}} V_{\lambda} g, V_{\lambda} g\right\rangle=\left\langle V_{\lambda}^{*} T V_{\lambda} \psi, \psi\right\rangle=\sqrt{2 \pi}\langle\hat{K}(M+\lambda) \hat{\psi}, \hat{\psi}\rangle \\
(2.19) & =\sqrt{2 \pi} \int \hat{K}(t+\lambda) d\left\langle E_{t} \hat{\psi}, \hat{\psi}\right\rangle,
\end{aligned}
$$

where $E_{t}$ is the spectral measure for the multiplication operator $(M f)(x)=x f(x)$. Conditions (2.5)-(2.7) ensure $\left|\hat{K}\left(t+\lambda_{j}+j\right)\right| \leq \sum_{k \geq j} 2^{-k}$ for $t \in[-j, j], j \in \mathbf{N}$, hence (2.18).

To prove (2.17), note that the sequences $\left\{\lambda_{j}\right\}_{j=0}^{\infty}$ and $\left\{m_{j}\right\}_{j=1}^{\infty}$ were defined so that $\left[2^{m_{j}} \omega-j, 2^{m_{j}} \omega+j\right] \subset\left(\lambda_{j-1}, \lambda_{j}\right)$. By $(2.5)-(2.7)$, if $\lambda_{j-1} \leq|\lambda| \leq \lambda_{j}$, then $\sum_{k \neq j}\left|\hat{\xi}_{m_{k}}(\lambda)\right| \leq \sum_{k \geq j} 2^{-k}=2^{-j+1}$. Therefore

$$
\left|\hat{K}\left(t+2^{m_{j}} \omega\right)-\hat{\xi}\left(2^{-m_{j}} t+\omega\right)\right|=\left|\hat{K}\left(t+2^{m_{j}} \omega\right)-\hat{\xi}_{m_{j}}\left(t+2^{m_{j}} \omega\right)\right| \leq 2^{-j+1}
$$

if $|t| \leq j$. Thus for any given $0<R<\infty$, if $j \geq R$, then

$$
\sup _{|t| \leq R}\left|\hat{K}\left(t+2^{m_{j}} \omega\right)-\hat{\xi}(\omega)\right| \leq 2^{-j+1}+\sup _{|t| \leq R}\left|\hat{\xi}\left(2^{-m_{j}} t+\omega\right)-\hat{\xi}(\omega)\right| .
$$


It now follows from $(2.19),(2.20)$ and the continuity of $\hat{\xi}$ that

$$
\lim _{j \rightarrow \infty}\left\langle\tilde{V}_{2^{m_{j}}{ }_{\omega}} B \tilde{V}_{2^{m_{j}} \omega} g, g\right\rangle=\sqrt{2 \pi} \hat{\xi}(\omega)\|\hat{\psi}\|^{2} .
$$

Since $g \in H^{2}(\mathbf{R})$ and $\|g\|>0, g$ cannot vanish on a set of positive measure in $\mathbf{R}$. Therefore $\|\hat{\psi}\|>0$. Since $\hat{\xi}(\omega) \neq 0$ (see (2.2)), this proves (2.17).

This completes the proof of Theorem 1.2.

\section{Proof of Theorem 1.4}

In this section we are back to the setting of the unit circle $\mathbf{T}=\{\tau \in \mathbf{C}:|\tau|=1\}$. Let $d m$ be the Lebesgue measure on $\mathbf{T}$ normalized so that $m(\mathbf{T})=1$. For an arc $I$ in $\mathbf{T}$, we write $|I|$ for $m(I)$. We will prove the following slightly more general result:

Proposition 3.1. Let $X \in \mathcal{B}\left(L^{2}\right)$ be an operator in the essential commutant of $\left\{M_{f}: f \in Q C\right\}$. Then for any $\epsilon>0$, there is a $\delta=\delta(\epsilon)>0$ such that the inequality $\left\|\left[M_{\varphi}, X\right]\right\| \leq \epsilon$ holds for every $\varphi \in L^{\infty}$ satisfying the conditions $\|\varphi\|_{B M O} \leq \delta$ and $\|\varphi\|_{\infty} \leq 1$.

Proof of Theorem 1.4. The "if" part of the theorem is trivial; it simply follows from the fact that for every $f \in \mathrm{QC}$, there is a sequence $\left\{f_{n}\right\} \subset C(\mathbf{T})$ such that $\left\|f_{n}\right\|_{\infty} \leq\|f\|_{\infty}$ and $\lim _{n \rightarrow \infty}\left\|f-f_{n}\right\|_{\mathrm{BMO}}=0$ [5, Theorem VI.5.1].

To prove the "only if" part of the theorem, consider an $A \in \operatorname{EssCom}(\mathcal{T}(\mathrm{QC}))$. Let $X=A \oplus 0$, where the direct sum corresponds to the space decomposition $L^{2}=H^{2} \oplus\left(H^{2}\right)^{\perp}$. Let $f \in \mathrm{QC}$. Then $P M_{f}(1-P)$ and $(1-P) M_{f} P$ are compact. Since $X=P X P$, we have

$$
\left[M_{f}, X\right]=(1-P) M_{f} P X P-P X P M_{f}(1-P)+\left[T_{f}, A\right] \oplus 0,
$$

which is compact because $A \in \operatorname{EssCom}(\mathcal{T}(\mathrm{QC}))$. Thus

$$
X \in \operatorname{Ess} \operatorname{Com}\left(\left\{M_{f}: f \in \mathrm{QC}\right\}\right) .
$$

Now for any $\varphi \in L^{\infty}$ we have $\left[T_{\varphi}, A\right] \oplus 0=P\left[M_{\varphi}, X\right] P$ and, therefore,

$$
\left\|\left[T_{\varphi}, A\right]\right\| \leq\left\|\left[M_{\varphi}, X\right]\right\| .
$$

The desired bound for $\left\|\left[T_{\varphi}, A\right]\right\|$ follows from an application of Proposition 3.1.

The proof of Proposition 3.1 requires some preparations. Our first lemma provides continuous cutoff functions with small BMO-norm.

Lemma 3.2. For any $0<\epsilon \leq 1 / 4$, there is a $0<\delta(\epsilon)<\epsilon$ such that the following holds true: Let $J=\left\{\tau e^{2 \pi i t}:|t|<\epsilon / 2\right\}$ and $I=\left\{\tau e^{2 \pi i t}:|t| \leq \delta(\epsilon) / 2\right\}$, where $\tau$ is any given point in $\mathbf{T}$. Then there is a $v \in C(\mathbf{T})$ which has the following properties:

(i) $0 \leq v \leq 1$ on $\mathbf{T}$;

(ii) $v=0$ on $\mathbf{T} \backslash J$;

(iii) $v=1$ on $I$;

(iv) $\|v\|_{B M O} \leq 4 \epsilon$.

Proof. Let $C$ be the BMO-norm of the function $\log (1 /|t|)$, which is finite [5, page 223]. For any $0<\epsilon \leq 1 / 4$, define $\delta(\epsilon)$ to be the real number that satisfies the equation

$$
\frac{1}{\log (2 / \delta(\epsilon))-\log (2 / \epsilon)}=\frac{\epsilon}{4 C}
$$


Thus $\delta(\epsilon)=\epsilon e^{-4 C / \epsilon}<\epsilon$. Define

$$
f(t)=\min \left\{\log \frac{2}{\delta(\epsilon)}, \max \left\{\log \frac{2}{\epsilon}, \log \frac{1}{|t|}\right\}\right\}-\log \frac{2}{\epsilon} .
$$

Obviously, $f$ is continuous, $0 \leq f \leq \log (2 / \delta(\epsilon))-\log (2 / \epsilon), f(t)=0$ when $|t| \geq \epsilon / 2$, and $f(t)=\log (2 / \delta(\epsilon))-\log (2 / \epsilon)$ when $|t| \leq \delta(\epsilon) / 2$.

For any real-valued $h \in \mathrm{BMO}(\mathbf{R})$ and $\lambda \in \mathbf{R}$, it follows from the formula $\max \{\lambda, h\}=2^{-1}(|\lambda-h|+\lambda+h)$ that $\|\max \{\lambda, h\}\|_{\text {BMO }} \leq 2\|h\|_{\text {BMO }}$. Similarly, we have

$$
\|\min \{\lambda, h\}\|_{\mathrm{BMO}} \leq 2\|h\|_{\mathrm{BMO}} .
$$

Hence $\|f\|_{\text {BMO }} \leq 4 C$. It is now easy to see that the function

$$
v\left(\tau e^{2 \pi i t}\right)=\frac{f(t)}{\log (2 / \delta(\epsilon))-\log (2 / \epsilon)}, \quad|t| \leq 1 / 2,
$$

is continuous on $\mathbf{T}$ and satisfies (i)-(iv).

Let $P_{z}(\tau)$ be the Poisson kernel for the unit disc, i.e.,

$$
P_{z}(\tau)=\frac{1-|z|^{2}}{|1-\bar{\tau} z|^{2}},
$$

$|z|<1,|\tau|=1$. For $\varphi \in L^{\infty}$ and $0 \leq r<1$, let

$$
\varphi_{r}\left(e^{2 \pi i t}\right)=\int P_{r}(\tau) \varphi\left(e^{2 \pi i t} \tau\right) d m(\tau),
$$

$t \in \mathbf{R}$. Our next lemma is most likely a well-known fact, but we include a proof here just in case it is not as well known as we think.

Lemma 3.3. For $\varphi \in L^{\infty}$ and $0 \leq r<1$ we have $\left\|\varphi_{r}\right\|_{B M O} \leq\|\varphi\|_{B M O}$.

Proof. For each $\tau \in \mathbf{T}$, define the rotation $\rho_{\tau}\left(e^{2 \pi i t}\right)=e^{2 \pi i t} \tau$ of the unit circle. Then $\varphi_{r}=\int P_{r}(\tau) \varphi \circ \rho_{\tau} d m(\tau)$. Thus for any arc $I$ in $\mathbf{T}$ we have

$$
\left(\varphi_{r}\right)_{I}=\int P_{r}(\tau)\left(\varphi \circ \rho_{\tau}\right)_{I} d m(\tau)
$$

and

$$
\begin{aligned}
\frac{1}{|I|} \int_{I}\left|\varphi_{r}-\left(\varphi_{r}\right)_{I}\right| d m & =\frac{1}{|I|} \int_{I}\left|\int P_{r}(\tau)\left(\varphi \circ \rho_{\tau}-\left(\varphi \circ \rho_{\tau}\right)_{I}\right) d m(\tau)\right| d m \\
& \leq \int P_{r}(\tau)\left\{\frac{1}{|I|} \int_{I}\left|\varphi \circ \rho_{\tau}-\left(\varphi \circ \rho_{\tau}\right)_{I}\right| d m\right\} d m(\tau) .
\end{aligned}
$$

Obviously, $\left\|\varphi \circ \rho_{\tau}\right\|_{\mathrm{BMO}}=\|\varphi\|_{\mathrm{BMO}}$ for every $\tau \in \mathbf{T}$. Hence $\left\|\varphi_{r}\right\|_{\mathrm{BMO}} \leq\|\varphi\|_{\mathrm{BMO}}$.

Our final lemma is a previously established result.

Lemma 3.4 ([9, Lemma 2.1]). Let $\left\{B_{n}\right\}$ be a sequence of compact operators on a Hilbert space $H$ satisfying the following two conditions:

(i) Both sequences $\left\{B_{n}\right\}$ and $\left\{B_{n}^{*}\right\}$ converge to 0 in the strong operator topology.

(ii) The limit $\lim _{n \rightarrow \infty}\left\|B_{n}\right\|$ exists.

Then there exists a subsequence $\{\mu(k)\}_{k=1}^{\infty}$ of $\{n\}_{n=1}^{\infty}$ such that the strong limit

$$
\sum_{k=1}^{\infty} B_{\mu(k)}=s-\lim _{N \rightarrow \infty} \sum_{k=1}^{N} B_{\mu(k)}
$$


exists and has the property

$$
\left\|\sum_{k=1}^{\infty} B_{\mu(k)}\right\|_{\mathcal{Q}}=\lim _{n \rightarrow \infty}\left\|B_{n}\right\|
$$

where $\|.\|_{\mathcal{Q}}$ denotes the essential norm, i.e., $\|A\|_{\mathcal{Q}}=\inf \{\|A+K\|: K \in \mathcal{K}(H)\}$.

Proof of Proposition 3.1. Let $Y$ be a bounded operator on $L^{2}$. Suppose that there exist an $a>0$ and a sequence $\left\{\varphi_{k}\right\} \subset L^{\infty}$ such that

$$
\left\|\left[M_{\varphi_{k}}, Y\right]\right\|>a \text { and }\left\|\varphi_{k}\right\|_{\infty} \leq 1
$$

for every $k$ and such that

$$
\lim _{k \rightarrow \infty}\left\|\varphi_{k}\right\|_{\mathrm{BMO}}=0 .
$$

We will complete the proof by finding an $f \in \mathrm{QC}$ such that $\left[M_{f}, Y\right]$ is not compact. We may assume that $\left[M_{g}, Y\right]$ is compact for every $g \in C(\mathbf{T})$, for otherwise there is no need to proceed any further.

For each $k$, we have $\left\|\left(\varphi_{k}\right)_{r}\right\|_{\infty} \leq\left\|\varphi_{k}\right\|_{\infty} \leq 1$ and, by Lemma 3.3, $\left\|\left(\varphi_{k}\right)_{r}\right\|_{\mathrm{BMO}} \leq$

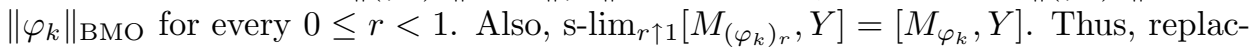
ing each $\varphi_{k}$ by an appropriate $\left(\varphi_{k}\right)_{r}$ if necessary, we may assume that the sequence $\left\{\varphi_{k}\right\}$ above is actually contained in $C(\mathbf{T})$.

We claim that for each $n \in \mathbf{N}$, there exist an arc $I_{n}$ with $\left|I_{n}\right| \leq 2 / n$ and a $\psi_{n} \in C(\mathbf{T})$ with $\left\|\psi_{n}\right\|_{\infty} \leq 1$ and $\left\|\psi_{n}\right\|_{\mathrm{BMO}} \leq 2^{-n}$ such that

$$
\left\|M_{\chi_{I_{n}}}\left[M_{\psi_{n}}, Y\right] M_{\chi_{I_{n}}}\right\| \geq a / 4 \text {. }
$$

For this purpose we consider the arcs $J_{j}=\left\{e^{2 \pi i t}:(j-1) / n \leq t<j / n\right\}, j=1,2$, $\ldots, n$. We further define $J_{0}=J_{n}$ and $J_{n+1}=J_{1}$. Thus

$\left[M_{\varphi_{k}}, Y\right]=\sum_{1 \leq j, j^{\prime} \leq n}\left[M_{\varphi_{k}}, M_{\chi_{j^{\prime}}} Y M_{\chi_{J_{j}}}\right]=\sum_{i=-1}^{1} \sum_{j=1}^{n}\left[M_{\varphi_{k}}, M_{\chi_{J_{j+i}}} Y M_{\chi_{J_{j}}}\right]+\left[M_{\varphi_{k}}, L\right]$,

where

$$
L=\sum_{\left|j^{\prime}-j\right| \geq 2} M_{\chi_{j^{\prime}}} Y M_{\chi_{J_{j}}}
$$

If $j \in\{1, \ldots, n\}$ and $\left|j^{\prime}-j\right| \geq 2$, then $d\left(J_{j^{\prime}}, J_{j}\right)>0$. Thus there are $g_{j^{\prime}}$, $g_{j} \in C(\mathbf{T})$ such that $g_{j^{\prime}}=1$ on $J_{j^{\prime}}, g_{j}=1$ on $J_{j}$, and $g_{j^{\prime}} g_{j}=0$. Since $g_{j} \in C(\mathbf{T})$, the operator $M_{g_{j^{\prime}}} Y M_{g_{j}}=M_{g_{j^{\prime}}}\left[Y, M_{g_{j}}\right]$ is compact by assumption. Thus $M_{\chi_{j^{\prime}}} Y M_{\chi_{J_{j}}}=M_{\chi_{J_{j^{\prime}}}} M_{g_{j^{\prime}}} Y M_{g_{j}} M_{\chi_{J_{j}}}$ is also compact. It follows that $L$ is a compact operator. Let $c_{k}=\int \varphi_{k} d m, k \in \mathbf{N}$. Then

$$
\lim _{k \rightarrow \infty} \int\left|\varphi_{k}-c_{k}\right| d m \leq \lim _{k \rightarrow \infty}\left\|\varphi_{k}\right\|_{\mathrm{BMO}}=0 .
$$

Since $\left\|\varphi_{k}\right\|_{\infty} \leq 1$, this implies $\mathrm{s}-\lim _{k \rightarrow \infty} M_{\varphi_{k}-c_{k}}=0$ and $\mathrm{s}-\lim _{k \rightarrow \infty} M_{\varphi_{k}-c_{k}}^{*}=0$. By the compactness of $L$, we have

$$
\lim _{k \rightarrow \infty}\left\|\left[M_{\varphi_{k}}, L\right]\right\|=\lim _{k \rightarrow \infty}\left\|\left[M_{\varphi_{k}-c_{k}}, L\right]\right\|=0 .
$$

By (3.1)-(3.2) and (3.4)-(3.5), there is a $k(n) \in \mathbf{N}$ such that $\left\|\varphi_{k(n)}\right\|_{\text {BMO }} \leq 2^{-n}$ and such that

$$
\left\|\sum_{i=-1}^{1} \sum_{j=1}^{n}\left[M_{\varphi_{k(n)}}, M_{\chi_{J_{j+i}}} Y M_{\chi_{J_{j}}}\right]\right\| \geq 3 a / 4
$$


We set $\psi_{n}=\varphi_{k(n)}$. Then $\left\|\psi_{n}\right\|_{\mathrm{BMO}} \leq 2^{-n}$ and $\left\|\psi_{n}\right\|_{\infty} \leq 1$. The above implies that there is an $i(n) \in\{-1,0,1\}$ such that

$$
\left\|\sum_{j=1}^{n} M_{\chi_{J_{j+i(n)}}}\left[M_{\psi_{n}}, Y\right] M_{\chi_{J_{j}}}\right\|=\left\|\sum_{j=1}^{n}\left[M_{\psi_{n}}, M_{\chi_{J_{j+i(n)}}} Y M_{\chi_{J_{j}}}\right]\right\| \geq a / 4 .
$$

Since $J_{1}, \ldots, J_{n}$ are disjoint, this implies that there is a $j(n) \in\{1, \ldots, n\}$ such that

$$
\left\|M_{\chi_{J_{j(n)+i(n)}}}\left[M_{\psi_{n}}, Y\right] M_{\chi_{J_{j}(n)}}\right\| \geq a / 4 .
$$

Thus (3.3) holds for $I_{n}=J_{j(n)+i(n)} \cup J_{j(n)}$, proving our claim.

For each $k \in \mathbf{N}$, let $\delta\left(2^{-k}\right)$ be the number provided by Lemma 3.2 for $\epsilon=2^{-k}$. Given a $k \in \mathbf{N}$, let $n(k) \in \mathbf{N}$ be such that $2 / n(k) \leq \delta\left(2^{-k}\right)$. (Since $\delta\left(2^{-k}\right) \leq 2^{-k}$, this implies $n(k) \geq 2^{k+1}$.) By Lemma 3.2, there is an open arc $Q_{k}$ containing $I_{n(k)}$ and a continuous function $0 \leq v_{k} \leq 1$ on $\mathbf{T}$ such that $\left|Q_{k}\right|=2^{-k}, v_{k}=1$ on $I_{n(k)}$, $v_{k}=0$ on $\mathbf{T} \backslash Q_{k}$, and $\left\|v_{k}\right\|_{\mathrm{BMO}} \leq 2^{-k+2}$. Define

$$
g_{k}=v_{k} \psi_{n(k)},
$$

$k \in \mathbf{N}$. Thus $g_{k} \in C(\mathbf{T}), g_{k}=0$ on $\mathbf{T} \backslash Q_{k}$ and $\left\|g_{k}\right\|_{\infty} \leq 1$. Since $\left|v_{k}\right| \leq 1$ and $\left|\psi_{n(k)}\right| \leq 1$, we have $\left|g_{k}-\left(v_{k}\right)_{I}\left(\psi_{n(k)}\right)_{I}\right| \leq\left|v_{k}-\left(v_{k}\right)_{I}\right|+\left|\psi_{n(k)}-\left(\psi_{n(k)}\right)_{I}\right|$. Hence

$$
\left\|g_{k}\right\|_{\mathrm{BMO}} \leq 2\left(\left\|v_{k}\right\|_{\mathrm{BMO}}+\left\|\psi_{n(k)}\right\|_{\mathrm{BMO}}\right) \leq 2\left(2^{-k+2}+2^{-n(k)}\right) \leq 2^{-k+4} .
$$

Note that $\chi_{I_{n(k)}} v_{k}=\chi_{I_{n(k)}}$. Therefore

$$
\begin{aligned}
M_{\chi_{I_{n(k)}}}\left[M_{g_{k}}, Y\right] M_{\chi_{I_{n(k)}}} & =M_{\chi_{I_{n(k)}}}\left[M_{\chi_{I_{n(k)}}} M_{g_{k}}, Y\right] M_{\chi_{I_{n(k)}}} \\
& =M_{\chi_{I_{n(k)}}}\left[M_{\chi_{I_{n(k)}}} M_{\psi_{n(k)}}, Y\right] M_{\chi_{I_{n(k)}}} \\
& =M_{\chi_{I_{n(k)}}}\left[M_{\psi_{n(k)}}, Y\right] M_{\chi_{I_{n(k)}}} .
\end{aligned}
$$

Combining this identity with (3.3), we have

$$
\left\|\left[M_{g_{k}}, Y\right]\right\| \geq a / 4
$$

for every $k \in \mathbf{N}$.

Passing to a subsequence of $\left\{Q_{k}\right\}$ if necessary, we may assume that there is a $\sigma \in \mathbf{T}$ such that

$$
\lim _{k \rightarrow \infty} \sup _{\tau \in Q_{k}}|\tau-\sigma|=0 .
$$

Define $U_{n}=\left\{\sigma e^{2 \pi i t}:|t|<2^{-n-1}\right\}, n \in \mathbf{N}$. By Lemma 3.2, for each $n \in \mathbf{N}$ there exist a $0<\delta_{n}<2^{-n}$ and a $w_{n} \in C(\mathbf{T})$ such that $0 \leq w_{n} \leq 1$ on $\mathbf{T}, w_{n}=0$ on $\mathbf{T} \backslash U_{n},\left\|w_{n}\right\|_{\mathrm{BMO}} \leq 2^{-n+2}$, and $w_{n}=1$ on $V_{n}$, where

$$
V_{n}=\left\{\sigma e^{2 \pi i t}:|t|<\delta_{n} / 2\right\} .
$$

Let $\eta_{n}=1-w_{n}, n \in \mathbf{N}$. Then

$$
\left\|\eta_{n}\right\|_{\mathrm{BMO}}=\left\|w_{n}\right\|_{\mathrm{BMO}} \leq 2^{-n+2} .
$$

Also, $\left\|\eta_{n}\right\|_{\infty} \leq 1, \eta_{n}=1$ on $\mathbf{T} \backslash U_{n}$, and $\eta_{n}=0$ on $V_{n}$. Since $\lim _{n \rightarrow \infty}\left|U_{n}\right|=0$, we have

$$
\text { s- } \lim _{n \rightarrow \infty} M_{\eta_{n}}=1 \text {. }
$$


Again, $\left\|g_{k} \eta_{n}\right\|_{\mathrm{BMO}} \leq 2\left(\left\|g_{k}\right\|_{\mathrm{BMO}}+\left\|\eta_{n}\right\|_{\mathrm{BMO}}\right)$. By (3.6)-(3.7) and (3.9)-(3.10), for each $k \in \mathbf{N}$ there is an $n_{k} \in \mathbf{N}$ such that if we set $f_{k}=g_{k} \eta_{n_{k}}$, then $\left\|f_{k}\right\|_{\text {BMO }} \leq$ $2^{-k+6}$ and

$$
\left\|\left[M_{f_{k}}, Y\right]\right\| \geq a / 8 .
$$

Obviously, $f_{k} \in C(\mathbf{T}),\left\|f_{k}\right\|_{\infty} \leq 1$, and $f_{k}=0$ on $\left(\mathbf{T} \backslash Q_{k}\right) \cup V_{n_{k}}$.

Because every $V_{n_{k}}$ is a neighborhood of $\sigma$ and because of (3.8), there is a subsequence $\{k(j)\}_{j=1}^{\infty}$ of $\{k\}_{k=1}^{\infty}$ such that $f_{k(i)} f_{k(j)}=0$ on $\mathbf{T}$ for all $i \neq j$. Since $f_{k} \in$ $C(\mathbf{T})$, the operator $\left[M_{f_{k}}, Y\right]$ is compact by assumption. Since $\lim _{k \rightarrow \infty}\left|Q_{k}\right|=0$, we have

$$
\text { s- } \lim _{k \rightarrow \infty}\left[M_{f_{k}}, Y\right]=0=\mathrm{s}-\lim _{k \rightarrow \infty}\left[M_{f_{k}}, Y\right]^{*} .
$$

Thus by (3.11) and Lemma 3.4, there is a subsequence $\{\nu(j)\}_{j=1}^{\infty}$ of $\{k(j)\}_{j=1}^{\infty}$ such that the strong limit

$$
\text { S- } \lim _{N \rightarrow \infty} \sum_{j=1}^{N}\left[M_{f_{\nu(j)}}, Y\right]=\sum_{j=1}^{\infty}\left[M_{f_{\nu(j)}}, Y\right]
$$

is not compact. Define $f=\sum_{j=1}^{\infty} f_{\nu(j)}$. Because $f_{\nu(i)} f_{\nu(j)}=0$ for all $i \neq j$, we have $\|f\|_{\infty}=\sup _{j}\left\|f_{\nu(j)}\right\|_{\infty} \leq 1$. Furthermore, because $f_{k} \in C(\mathbf{T})$ and $\sum_{k=1}^{\infty}\left\|f_{k}\right\|_{\text {BMO }}<\infty$, we have $f \in$ VMO. Hence $f \in L^{\infty} \cap \mathrm{VMO}=\mathrm{QC}$. It is obvious that

$$
\left[M_{f}, Y\right]=\sum_{j=1}^{\infty}\left[M_{f_{\nu(j)}}, Y\right],
$$

which is not compact. This completes the proof.

\section{A REMARK ON TheOREM 1.4}

For the "if" part of Theorem 1.4, there are two assumptions on the operator $A$ :

(1) $\left[T_{h}, A\right]$ is compact for every continuous function $h$ on the unit circle.

(2) For every $\epsilon>0$, there exists a $\delta=\delta(\epsilon)>0$ such that the inequality $\left\|\left[T_{\varphi}, A\right]\right\| \leq \epsilon$ holds for every $\varphi \in L^{\infty}$ satisfying the conditions $\|\varphi\|_{\text {BMO }} \leq \delta$ and $\|\varphi\|_{\infty} \leq 1$.

A moment of reflection on these conditions would bring up the suspicion that (1) might be redundant. In other words, one would natually ask, does (2) imply (1)? If (1) could be dropped as an assumption, it would make the statement of Theorem 1.4 more pleasing. But the fact is that (2) does not imply (1), and therefore (1) cannot be dropped. In the remainder of the section we give an example to show that (2) does not imply (1).

To construct such an example, we use the John-Nirenberg Theorem again. By [5, Corollary VI.2.3], there is a universal constant $C_{4}$ such that the inequality

$$
\left\|\varphi-\varphi_{\mathbf{T}}\right\|_{4} \leq C_{4}\|\varphi\|_{\mathrm{BMO}}
$$

holds for every $\varphi \in L^{\infty}$, where $\varphi_{\mathbf{T}}=\int \varphi d m$ and $\|.\|_{4}$ denotes the $L^{4}$-norm.

For each integer $n \geq 0$, let $e_{n}(\tau)=\tau^{n}, \tau \in \mathbf{T}$. Let $\mathcal{E}$ be the norm closure of $\operatorname{span}\left\{e_{2^{k}}: k \in \mathbf{N}\right\}$ in $H^{2}$ and let $E: H^{2} \rightarrow \mathcal{E}$ be the orthogonal projection. It is obvious that

$$
\left[T_{e_{1}}, E\right] e_{2^{k}}=e_{1+2^{k}}, \quad k \in \mathbf{N} .
$$


Thus the operator $\left[T_{e_{1}}, E\right]$ is not compact. We will complete the example by showing that $E$ satisfies (2). In fact we will do a little better; we will show that the inequality

$$
\left\|\left[T_{\varphi}, E\right]\right\| \leq 2^{5 / 4} C_{4}\|\varphi\|_{\mathrm{BMO}}
$$

holds for every $\varphi \in L^{\infty}$.

To prove (4.2), consider $k, \ell, k^{\prime}, \ell^{\prime} \in \mathbf{N}$ satisfying the conditions $k \leq \ell$ and $k^{\prime} \leq \ell^{\prime}$. For such $k, \ell, k^{\prime}, \ell^{\prime}$, it is obvious that

$$
2^{k}+2^{\ell} \neq 2^{k^{\prime}}+2^{\ell^{\prime}} \text { if either } \ell \neq \ell^{\prime} \text { or } k \neq k^{\prime} .
$$

For any polynomial $p=\sum_{k=1}^{N} c_{k} e_{2^{k}}$ in $\mathcal{E}$, we have

$$
p^{2}=\sum_{k=1}^{N} c_{k}^{2} e_{2^{k}+2^{k}}+\sum_{1 \leq k<\ell \leq N} 2 c_{k} c_{\ell} e_{2^{k}+2^{\ell}} .
$$

By (4.3), we have $e_{2^{k}+2^{\ell}} \perp e_{2^{k^{\prime}}+2^{\ell^{\prime}}}$ under the conditions $(k, \ell) \neq\left(k^{\prime}, \ell^{\prime}\right), k, \ell, k^{\prime}, \ell^{\prime} \in$ $\mathbf{N}, k \leq \ell$ and $k^{\prime} \leq \ell^{\prime}$. Thus

$$
\|p\|_{4}^{4}=\left\langle p^{2}, p^{2}\right\rangle=\sum_{k=1}^{N}\left|c_{k}^{2}\right|^{2}+\sum_{1 \leq k<\ell \leq N} 4\left|c_{k} c_{\ell}\right|^{2} \leq 2\left(\sum_{k=1}^{N}\left|c_{k}\right|^{2}\right)^{2}=2\|p\|_{2}^{4} .
$$

Let $\varphi \in L^{\infty}$. If $q$ is a polynomial in $H^{2}$, then $E q$ is a polynomial in $\mathcal{E}$. We have

$$
\left\|T_{\varphi-\varphi_{\mathbf{T}}} E q\right\|_{2} \leq\left(\int\left|\left(\varphi-\varphi_{\mathbf{T}}\right) E q\right|^{2} d m\right)^{1 / 2} \leq\left\|\varphi-\varphi_{\mathbf{T}}\right\|_{4}\|E q\|_{4} .
$$

Applying (4.1) to $\varphi$ and (4.4) to $E q$, we have

$$
\left\|T_{\varphi-\varphi_{\mathrm{T}}} E q\right\|_{2} \leq C_{4}\|\varphi\|_{\mathrm{BMO}} \cdot 2^{1 / 4}\|E q\|_{2} \leq 2^{1 / 4} C_{4}\|\varphi\|_{\mathrm{BMO}}\|q\|_{2} .
$$

Since polynomials are dense in $H^{2}$, this implies that

$$
\left\|T_{\varphi-\varphi_{\mathrm{T}}} E\right\| \leq 2^{1 / 4} C_{4}\|\varphi\|_{\mathrm{BMO}},
$$

$\varphi \in L^{\infty}$. Now (4.2) follows from (4.5) and the identities $\left[T_{\varphi}, E\right]=T_{\varphi-\varphi_{\mathbf{T}}} E-$ $E T_{\varphi-\varphi_{\mathbf{T}}}$ and $\left\|E T_{\varphi-\varphi_{\mathbf{T}}}\right\|=\left\|\left(E T_{\varphi-\varphi_{\mathbf{T}}}\right)^{*}\right\|=\left\|T_{\bar{\varphi}-\bar{\varphi}_{\mathbf{T}}} E\right\|$.

\section{REFERENCES}

1. C. Berger and L. Coburn, On Voiculescu's double commutant theorem, Proc. Amer. Math. Soc. 124 (1996), 3453-3457. MR1346963 (97a:46067)

2. R. Coifman and C. Fefferman, Weighted norm inequalities for maximal functions and singular integrals, Studia Math. 51 (1974), 241-250. MR0358205 (50:10670)

3. R. Coifman, R. Rochberg and G. Weiss, Factorization theorems for Hardy spaces in several variables, Ann. of Math. 103 (1976), 611-635. MR0412721 (54:843)

4. K. Davidson, On operators commuting with Toeplitz operators modulo the compact operators, J. Funct. Anal. 24 (1977), 291-302. MR0454715 (56:12963)

5. J. Garnett, Bounded analytic functions, Academic Press, New York-London, 1981. MR628971 (83g:30037)

6. I. Gohberg and M. Krein, Introduction to the theory of linear nonselfadjoint operators, Amer. Math. Soc. Translations of Mathematical Monographs 18, Providence, 1969. MR0246142 (39:7447)

7. B. Johnson and S. Parrott, Operators commuting with a von Neumann algebra modulo the set of compact operators, J. Funct. Anal. 11 (1972), 39-61. MR0341119 (49:5869)

8. B. Muckenhoupt, Weighted norm inequalities for the Hardy maximal function, Trans. Amer. Math. Soc. 165 (1972), 207-226. MR0293384 (45:2461)

9. P. Muhly and J. Xia, On automorphisms of the Toeplitz algebra, Amer. J. Math. 122 (2000), 1121-1138. MR1797658 (2001m:46139) 
10. J. Orobitg and C. Pérez, $A_{p}$ weights for nondoubling measures in $R^{n}$ and applications, Trans. Amer. Math. Soc. 354 (2002), 2013-2033. MR1881028 (2002k:42044)

11. S. Popa, The commutant modulo the set of compact operators of a von Neumann algebra, J. Funct. Anal. 71 (1987), 393-408. MR880987 (88b:46091)

12. E. Stein, Singular integrals and differentiability properties of functions, Princeton Mathematical Series, 30, Princeton University Press, Princeton, 1970. MR0290095 (44:7280)

13. D. Voiculescu, A non-commutative Weyl-von Neumann theorem, Rev. Roumaine Math. Pures Appl. 21 (1976), 97-113. MR0415338 (54:3427)

14. J. Xia, Joint mean oscillation and local ideals in the Toeplitz algebra. II. Local commutivity and essential commutant, Canad. Math. Bull. 45 (2002), 309-318. MR1904095 (2003g:47052)

15. J. Xia, Coincidence of essential commutant and the double commutant relation in the Calkin algebra, J. Funct. Anal. 197 (2003), 140-150. MR1957677 (2003m:46092)

Department of Mathematics, State University of New York at Buffalo, Buffalo, New York 14260

E-mail address: jxia@acsu.buffalo.edu 